\title{
Parametric Study of Pulse-Combustor-Driven Ejectors at High-Pressure
}

\author{
Shaye Yungster \\ Ohio Aerospace Institute \\ Daniel E. Paxson and Hugh D. Perkins \\ NASA Glenn Research Center
}

AIAA Propulsion and Energy 2015

27 - 29 July 2015, Orlando, Florida 


\section{OAI}

\section{Overview}

- Objectives

- Investigate the performance characteristics of shrouded pulse-combustor configurations at high pressure conditions.

- The goal is to design configurations that maximize pressure gain while achieving a thermal environment acceptable to a turbine, and maintain acceptable levels of NOx emissions and flow non-uniformities

- Approach

- Utilize new computational platform, developed in previous studies, for studying pulse-combustors. 


\section{Introduction}

- Conventional gas turbine engine combustors based on steady, constant pressure combustion incur total pressure losses that can range from $4 \%$ to $8 \%$.

- Pressure-gain concepts:

- Pulse Detonation-Based devices

- Wave Rotors

- Pulse-combustors

- Pulse-combustors are unsteady, resonant thermo-acoustic devices in which heat released by combustion is coupled with the acoustic field.

- Experiments at atmospheric conditions demonstrated pressure gain of $\sim 3.5 \%$ (Paxson and Dougherty 2005).

- Preliminary CFD calculations at high-pressure conditions demonstrated pressure gain of $\sim 1.2 \%$ (Yungster et al. 2013).

- Maximum theoretical pressure-gains estimated at 7\% (Kentfield 1993). 


\section{OAI}

\section{Introduction}

- Advantages of Pulse-combustors over alternative pressure gain concepts:

- Avoids the mechanical complexities of higher pressure gain concepts.

- Pulse combustors are known to produce low NOx.

- Flow non-uniformities at exit of pulse-combustor are substantially reduced.

- Disadvantages of Pulse-combustors:

- Pressure-gains attainable are typically lower than those for wave rotors or detonation based devices (which can reach up 35\%). 
- Most previous studies of pulse-combustors have been carried out at atmospheric conditions.

- Practical aerospace applications of pressure-gain combustion systems necessitates operation at high-pressure conditions.

- Previous study (Yungster, Paxson and Perkins, 2013) analyzed differences in the operation of pulse-combustors at atmospheric $\left(p_{0}=1\right.$ bar; $\left.T_{0}=298 \mathrm{~K}\right)$ and highpressure conditions $\left(p_{0}=10 \mathrm{bar} ; T_{0}=550 \mathrm{~K}\right)$.

- Higher air temperature and pressure $\Rightarrow$ shorter ignition delay times

$>$ Change in combustion dynamics.

$>$ Increased operating frequency.

$>$ Necessitates fuel valving (to prevent pre-ignition).

$>$ Lower performance (pressure gain $\sim 1.2 \%$ ) 


\section{OAI}

\section{Introduction}

- A recent study (Yungster, Paxson and Perkins, 2014) identified the factors limiting the pressure-gain at high-pressure conditions.

- New pulse-combustor configurations were developed which were able to achieve performance levels at high-pressure conditions comparable to those observed at atmospheric conditions.

- However, suboptimal fuel distribution within the pulse-combustor was still limiting performance.

- The pulse-combustor by itself is not suitable to replace a conventional combustor in a gas turbine engine, and must be shrouded and combined with an ejector. 
Pulse-combustor device used in experiments

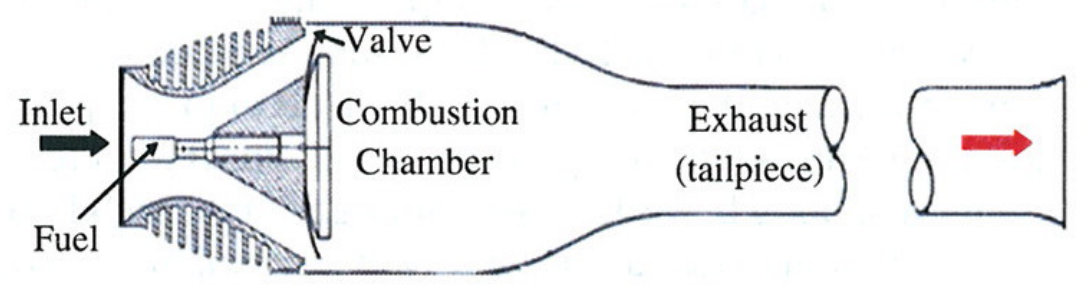

Computational model

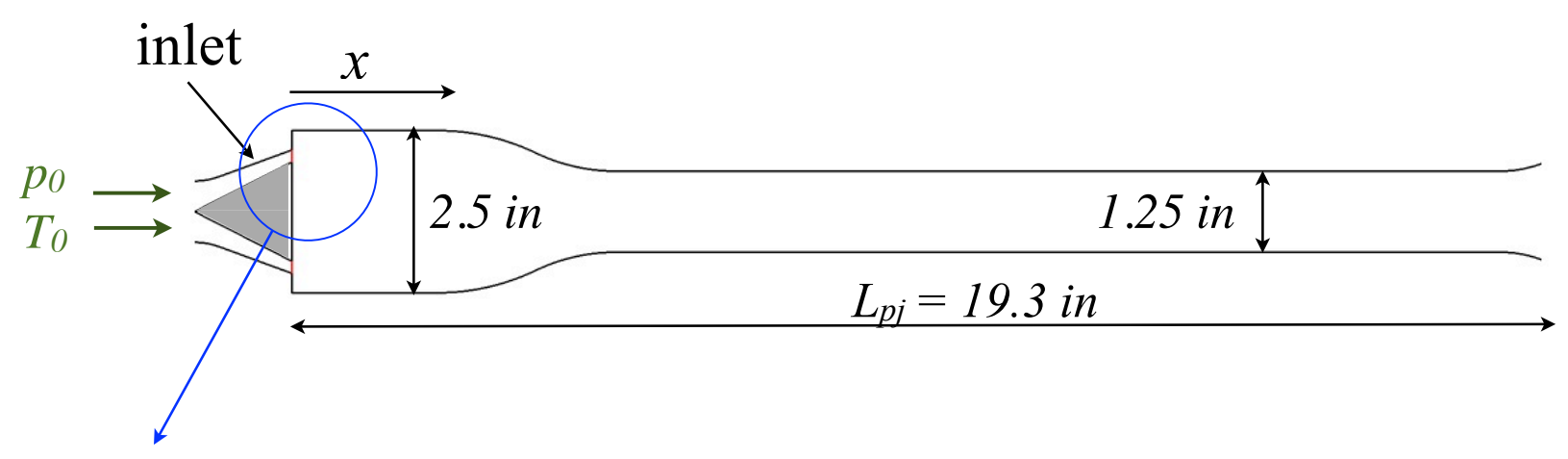

Valve (fully closed position)

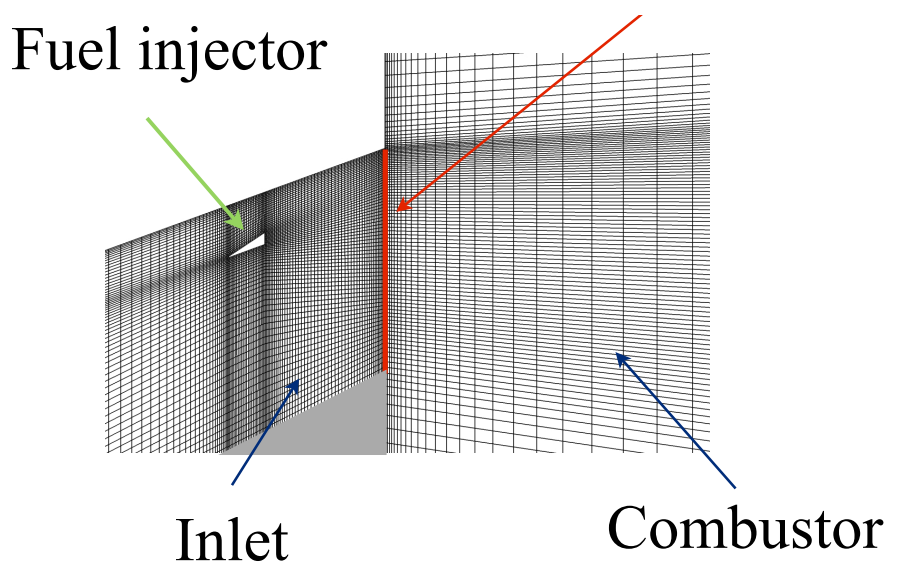

Valve (fully open position)

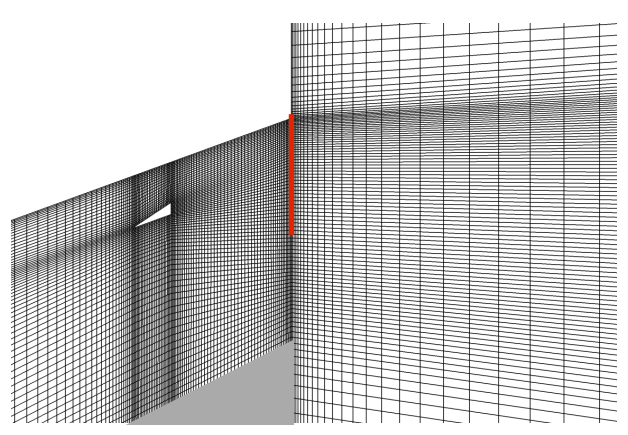

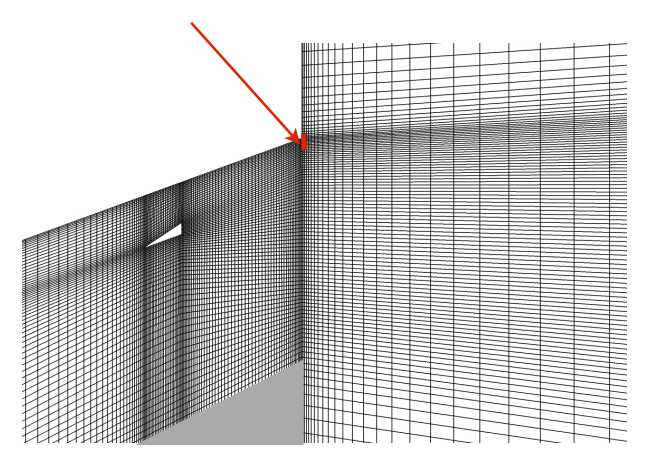


compression wave Ideal wave diagram

expansion wave

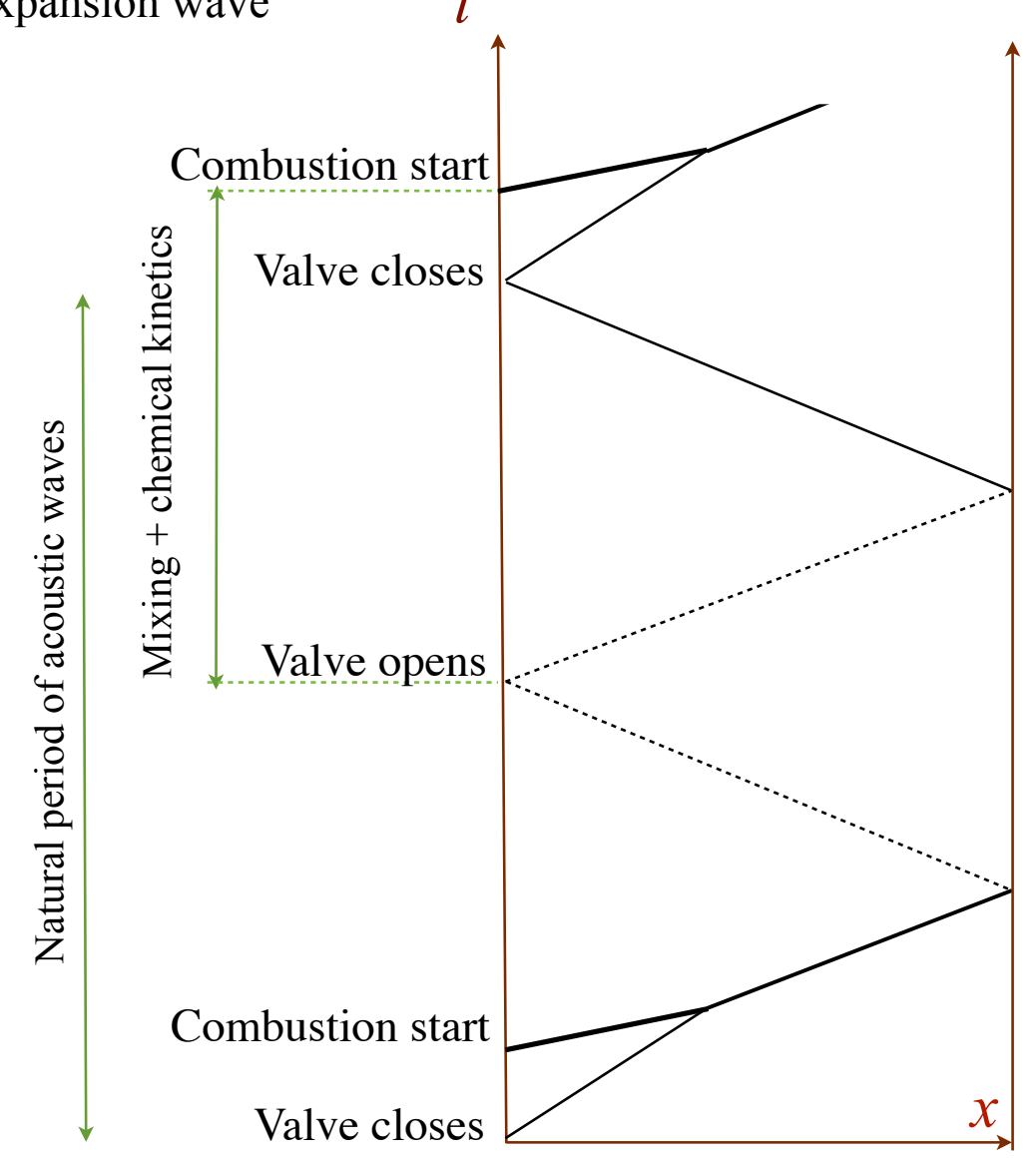

CFD simulation

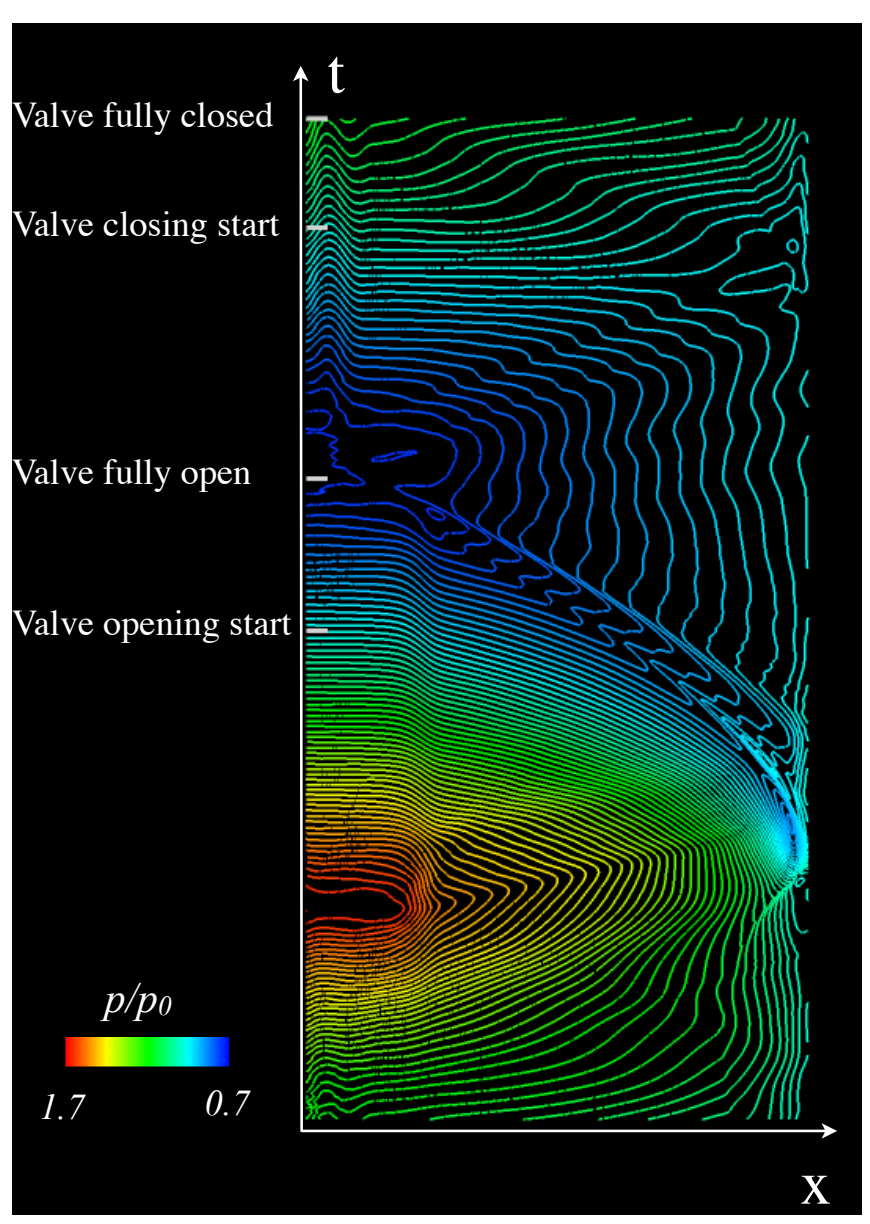




\section{Pulse-Combustor Pressure Variation}

(Yungster, Paxson and Perkins, 2013, 2014)

Fuel used in experiments: liquid gasoline Fuel used in CFD: gaseous jet-A

$$
p_{0}=1 \mathrm{bar}, T_{0}=298 \mathrm{~K}
$$

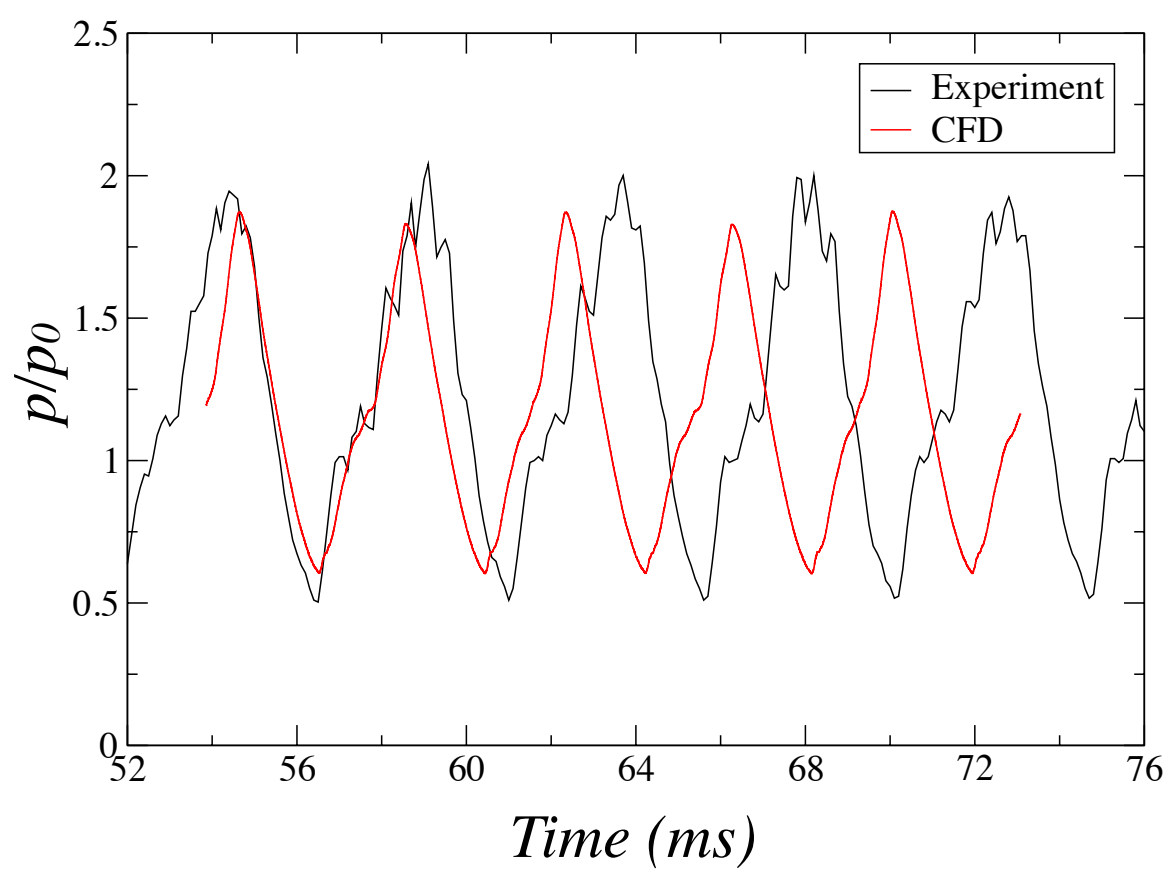

$$
f_{\text {exp }}=222 \mathrm{~Hz} ; f_{c f d}=255 \mathrm{~Hz}
$$$$
p_{\max } / p_{0}=1.87 ; p_{\text {avg }} / p_{0}=1.16
$$

CFD $\quad p_{0}=10 \mathrm{bar}, T_{0}=550 \mathrm{~K}$
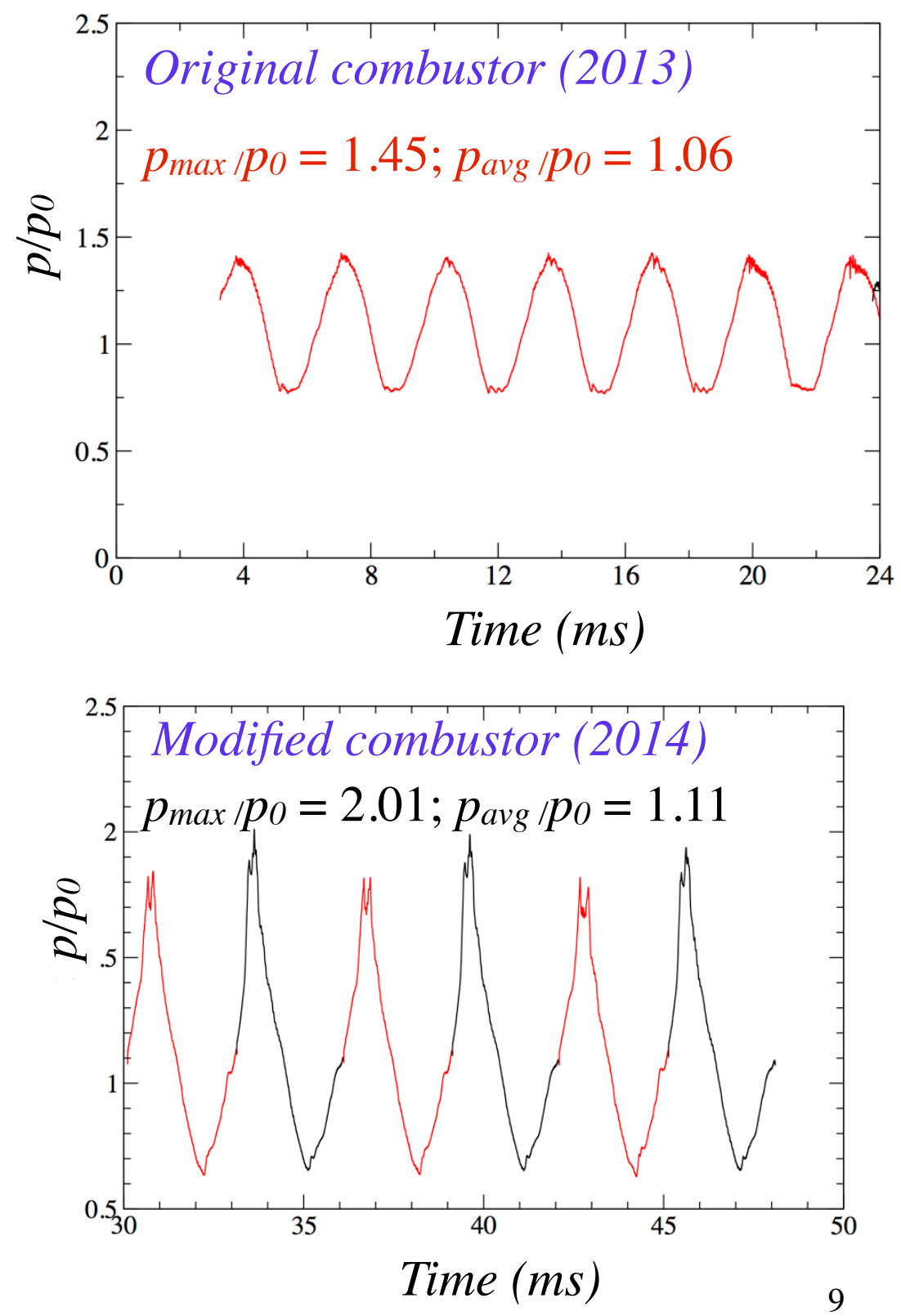
- In-house developed CFD code.

- (Yungster, S. and Radhakrishnan, K., "Pulsating One-Dimensional Detonations in Hydrogen-Air Mixtures," Combustion Theory and Modelling, 8, 745-770, 2004).

- Axisymmetric Navier-Stokes Equations for multi-species, thermally perfect, chemically reacting gas.

- Detailed chemistry capability

- Kundu's jet-A/air reaction mechanism (14-steps, 13-species). (has been successfully used in detonation and LDI combustor studies).

- Second-order TVD differencing scheme.

- Fully implicit BDF time marching algorithm.

- Spallart-Allmaras one-equation turbulence model.

\section{Approach}

- Conduct numerical simulations of the pulsejet-based devices for multiple cycles until limit-cycle operation is reached (8-25 cycles). 


\begin{tabular}{|c|c|c|c|c|}
\hline \multicolumn{5}{|c|}{ Jet-A Reaction Mechanism $^{\dagger}$} \\
\hline $\begin{array}{c}\text { No } \\
.\end{array}$ & Reaction & A & n & $\mathrm{E}^{\dagger \dagger}$ \\
\hline 1 & $\mathrm{C}_{11} \mathrm{H}_{21}+\mathrm{O}_{2} \Longrightarrow 11 \mathrm{CH}+10 \mathrm{H}+\mathrm{O}_{2}$ & $1.00 \times 10^{12}$ & 0 & $3.75 \times 10^{4}$ \\
\hline
\end{tabular}

forward $/ \mathrm{C}_{11} \mathrm{H}_{21} 0.8 /$; forward $/ \mathrm{O}_{2} \quad 0.8 /$

\begin{tabular}{|l|l|l|l|l|}
\hline 2 & $\mathrm{CH}+\mathrm{O}_{2} \Longleftrightarrow \mathrm{CO}+\mathrm{OH}$ & $2.00 \times 10^{15}$ & 0.00 & $3.00 \times 10^{3}$ \\
\hline 3 & $\mathrm{CH}+\mathrm{O} \Longleftrightarrow \mathrm{CO}+\mathrm{H}$ & $3.00 \times 10^{12}$ & 1.00 & 0.0 \\
\hline 4 & $\mathrm{H}_{2}+\mathrm{O}_{2} \Longleftrightarrow \mathrm{H}_{2} \mathrm{O}+\mathrm{O}$ & $3.98 \times 10^{11}$ & 1.00 & $4.80 \times 10^{4}$ \\
\hline 5 & $\mathrm{H}_{2}+\mathrm{O} \Longleftrightarrow \mathrm{H}+\mathrm{OH}$ & $3.00 \times 10^{14}$ & 0.00 & $6.00 \times 10^{3}$ \\
\hline 6 & $\mathrm{H}+\mathrm{O}_{2} \Longleftrightarrow \mathrm{O}+\mathrm{OH}$ & $4.00 \times 10^{14}$ & 0.00 & $1.80 \times 10^{4}$ \\
\hline 7 & $\mathrm{H}_{2} \mathrm{O}+\mathrm{O}_{2} \Longleftrightarrow \mathrm{H}_{2} \mathrm{O}+2 \mathrm{O}$ & $3.17 \times 10^{12}$ & 2.00 & $1.12 \times 10^{5}$ \\
\hline 8 & $\mathrm{CO}+\mathrm{OH} \Longleftrightarrow \mathrm{CO}_{2}+\mathrm{H}$ & $5.51 \times 10^{7}$ & 1.27 & $-7.58 \times 10^{2}$ \\
\hline 9 & $\mathrm{CO}+\mathrm{H}_{2} \mathrm{O} \Longleftrightarrow \mathrm{CO}_{2}+\mathrm{H}_{2}$ & $5.50 \times 10^{4}$ & 1.28 & $-1.00 \times 10^{3}$ \\
\hline 10 & $\mathrm{CO}+\mathrm{H}_{2}+\mathrm{O}_{2} \Longleftrightarrow \mathrm{CO}_{2}+\mathrm{H}_{2} \mathrm{O}$ & $1.60 \times 10^{14}$ & 1.60 & $1.80 \times 10^{4}$ \\
\hline 11 & $\mathrm{~N}+\mathrm{N}+\mathrm{M} \Longleftrightarrow \mathrm{N}_{2}+\mathrm{M}$ & $2.80 \times 10^{17}$ & -0.75 & 0.0 \\
\hline 12 & $\mathrm{~N}+\mathrm{O}_{2} \Longleftrightarrow \mathrm{NO}+\mathrm{O}$ & $6.40 \times 10^{9}$ & 1.00 & $6.30 \times 10^{3}$ \\
\hline 13 & $\mathrm{~N}+\mathrm{NO} \Longleftrightarrow \mathrm{N}_{2}+\mathrm{O}$ & $1.60 \times 10^{13}$ & 0.00 & 0.0 \\
\hline 14 & $\mathrm{~N}+\mathrm{OH} \Longleftrightarrow \mathrm{NO}+\mathrm{H}$ & $6.30 \times 10^{11}$ & 0.50 & 0.0 \\
\hline & & &
\end{tabular}

${ }^{\dagger}$ Forward rate coefficient ; units are moles, seconds, centimeters, calories and Kelvins. 


\section{Modified Pulse-Combustor and Axisymmetric Computational Domain.}

original combustor
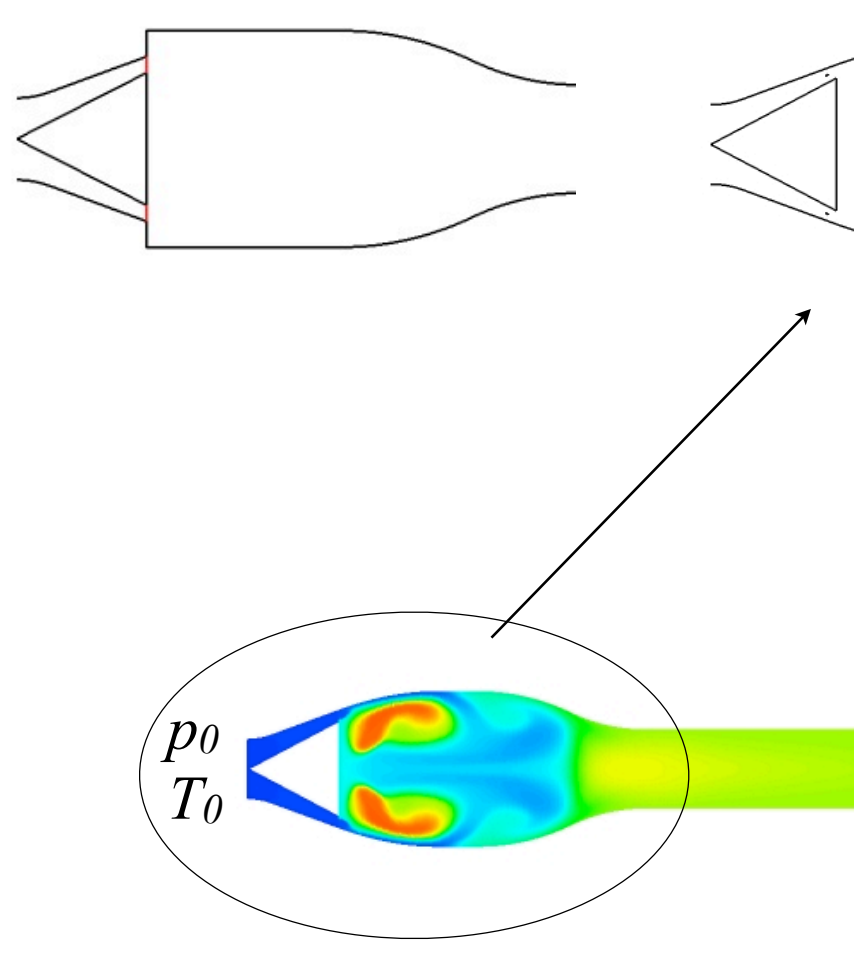

modified combustor
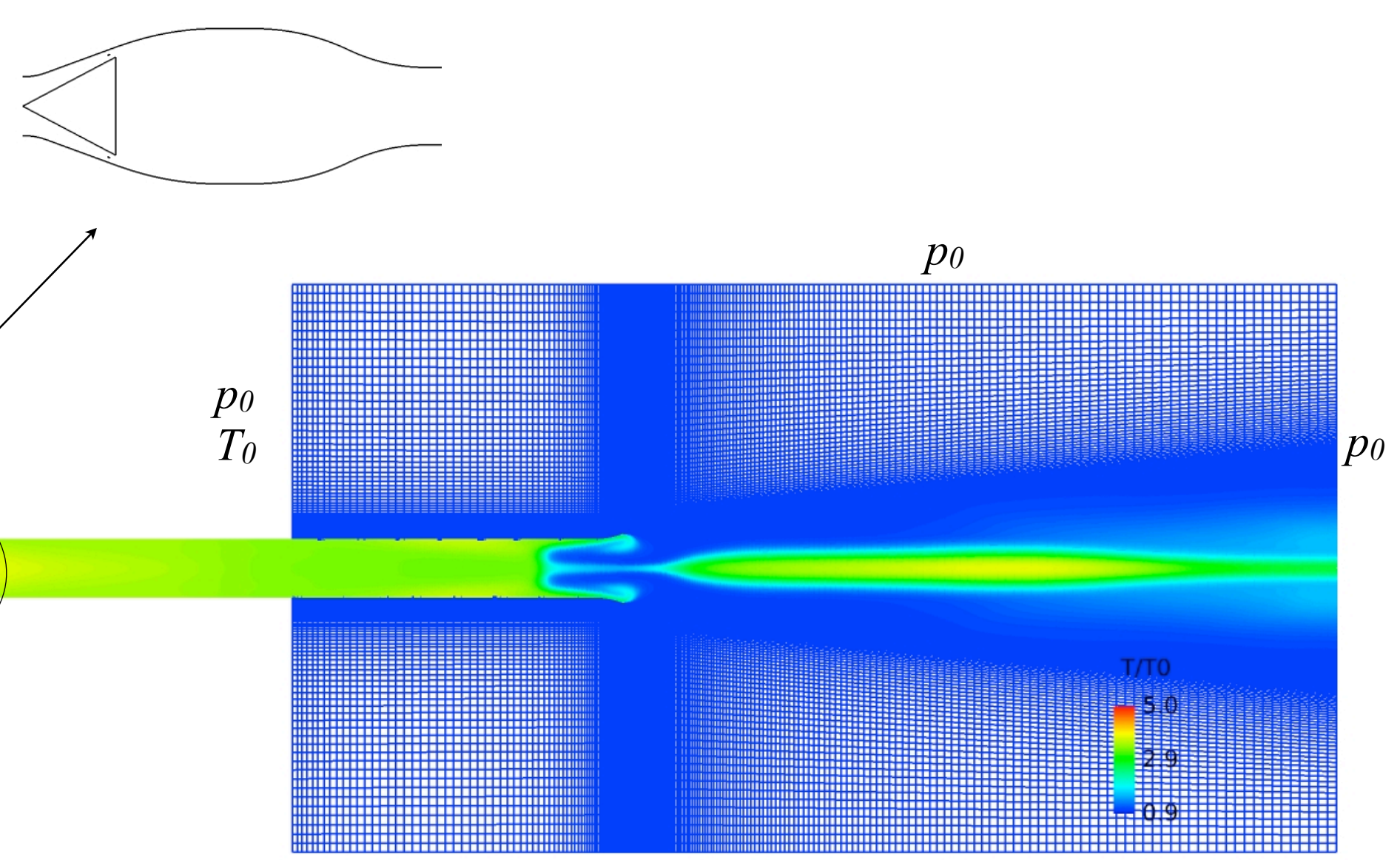


\section{OAI Pulse-Combustor Simulations at High-Pressure}

Baseline configuration

$p_{0}=10 \mathrm{bar}, T_{0}=550 \mathrm{~K}, \Phi=0.66$

$f=325 \mathrm{~Hz}$

$$
T / T_{0} \begin{aligned}
& 5.0 \\
& 2.9 \\
& 0.8
\end{aligned}
$$




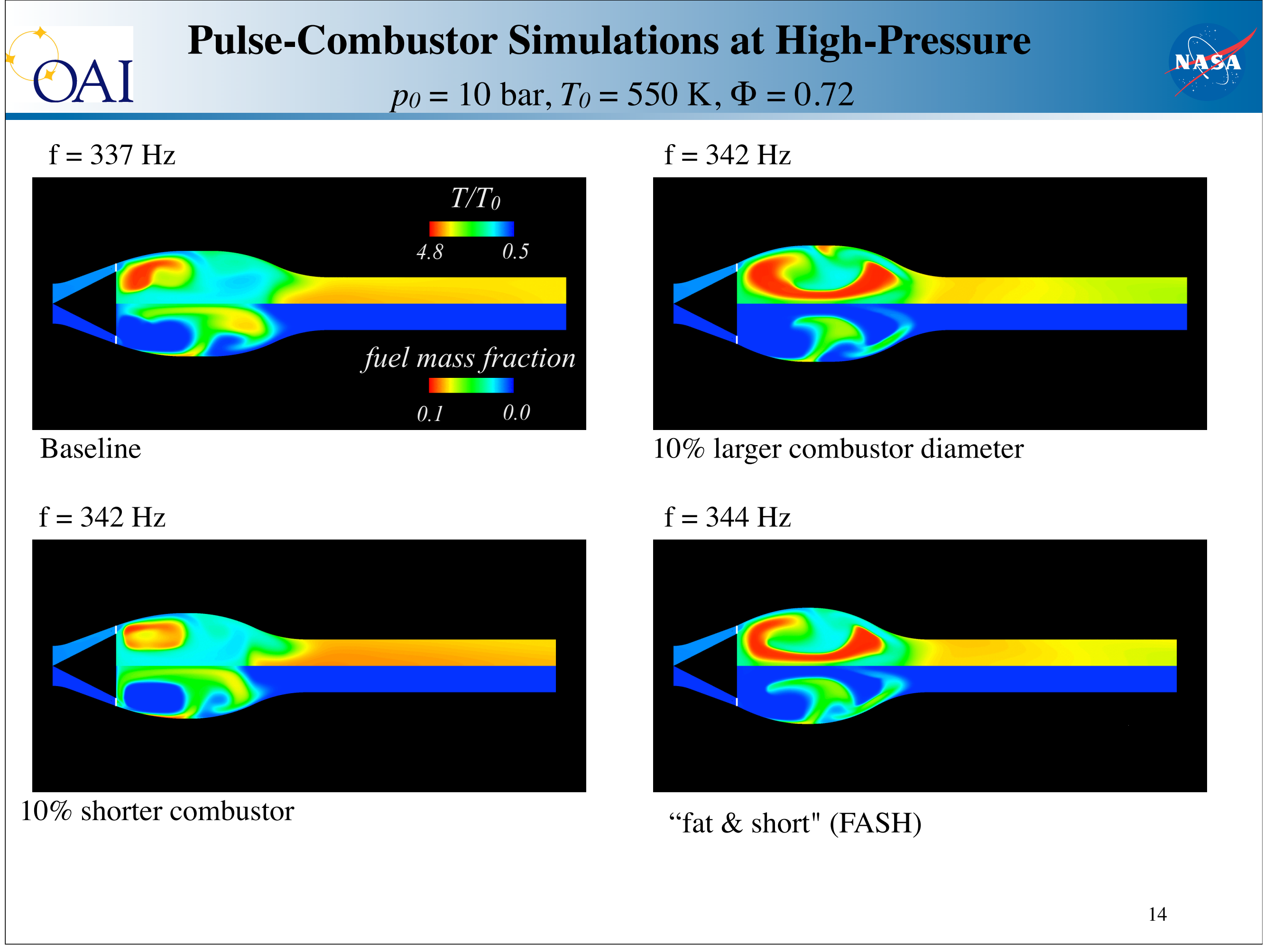




$$
p_{0}=10 \mathrm{bar}, T_{0}=550 \mathrm{~K}, \Phi=0.72
$$

primary vortex secondary vortex
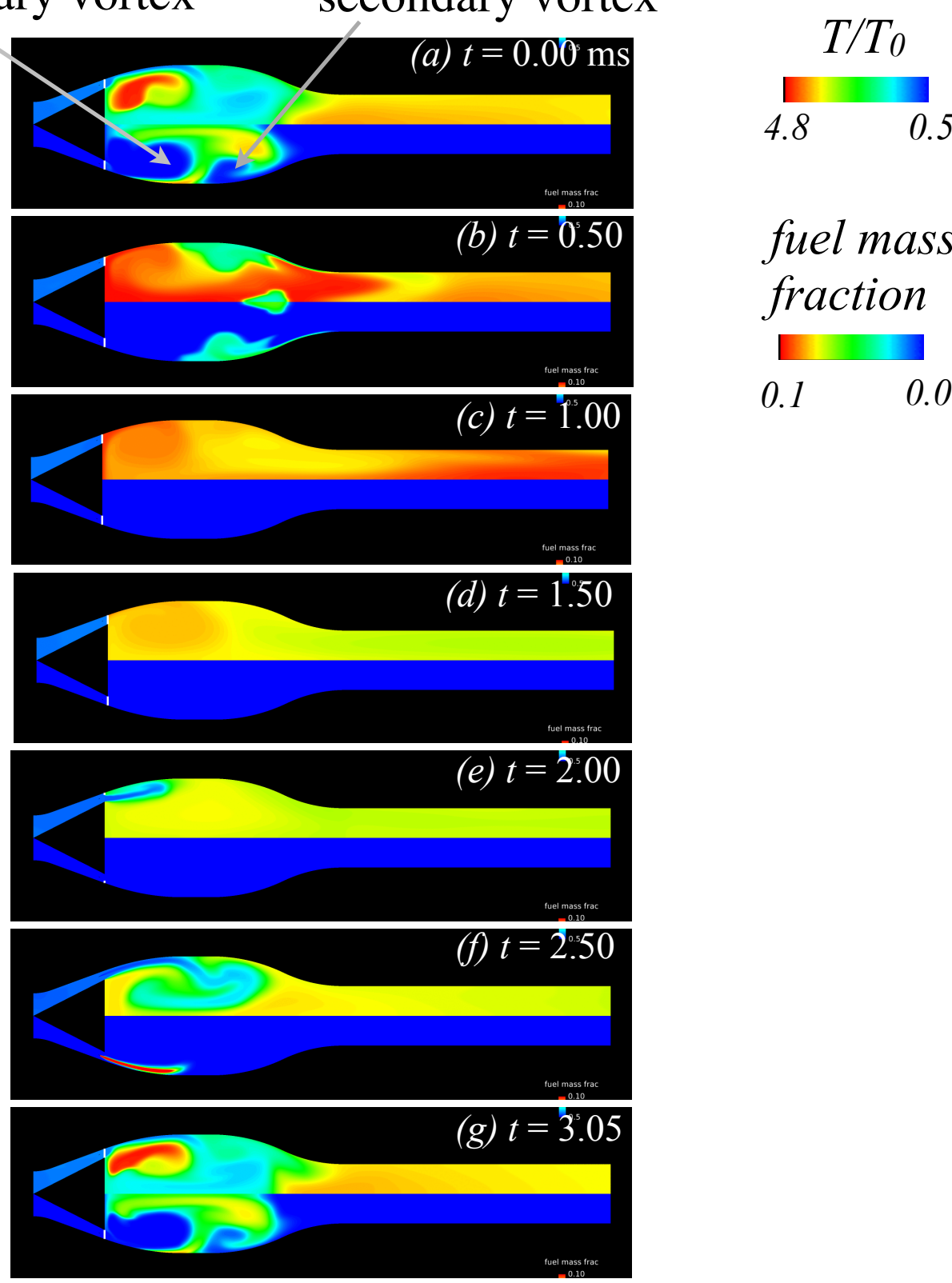

a) $t=0.00^{5} \mathrm{~ms}$

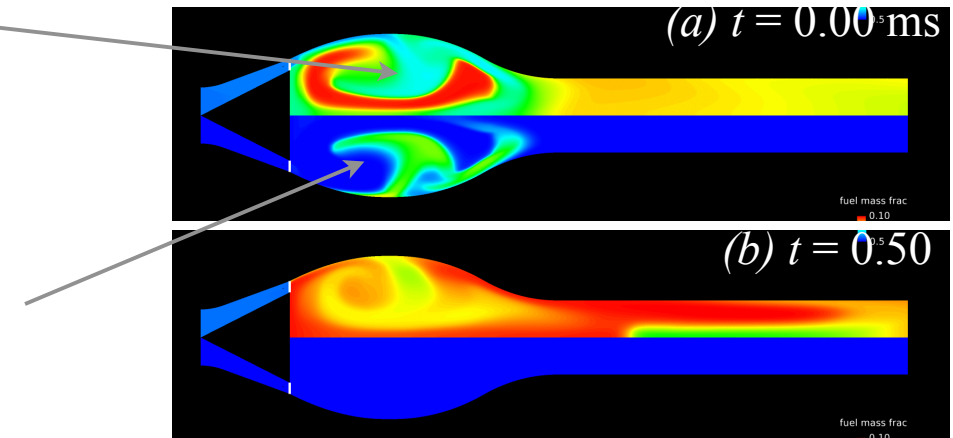

(c) $t=1.00$
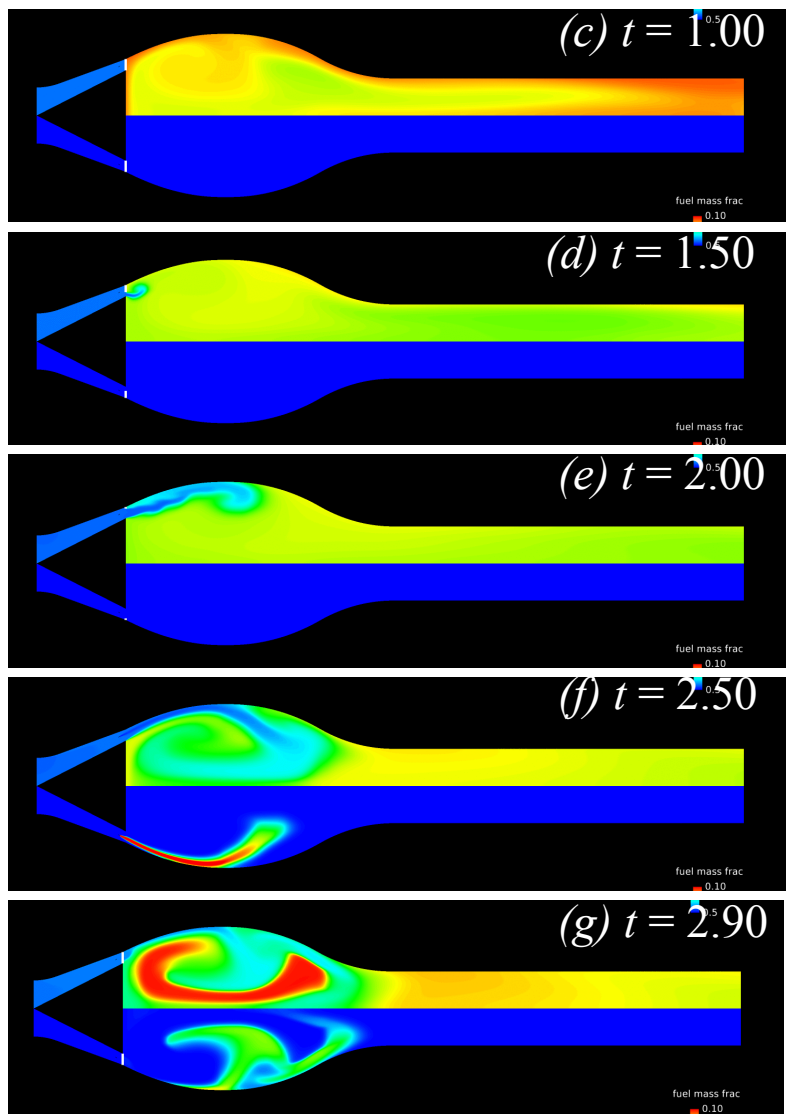

Baseline combustor

FASH combustor 


\section{Pulse-Combustor Simulations at High-Pressure}

$$
p_{0}=10 \mathrm{bar}, T_{0}=550 \mathrm{~K}, \Phi=0.72
$$

Baseline
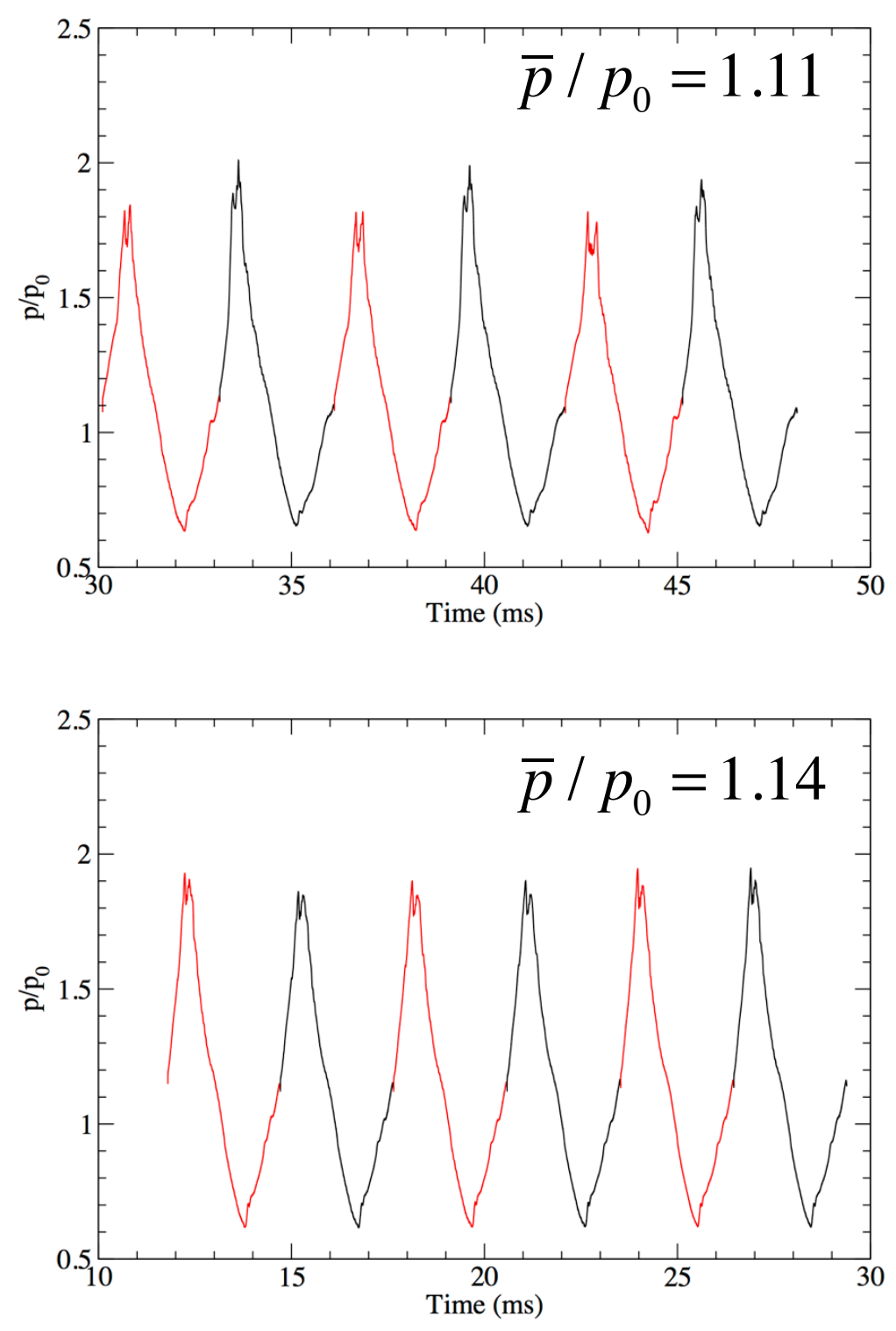

$10 \%$ shorter combustor
$10 \%$ larger combustor diameter
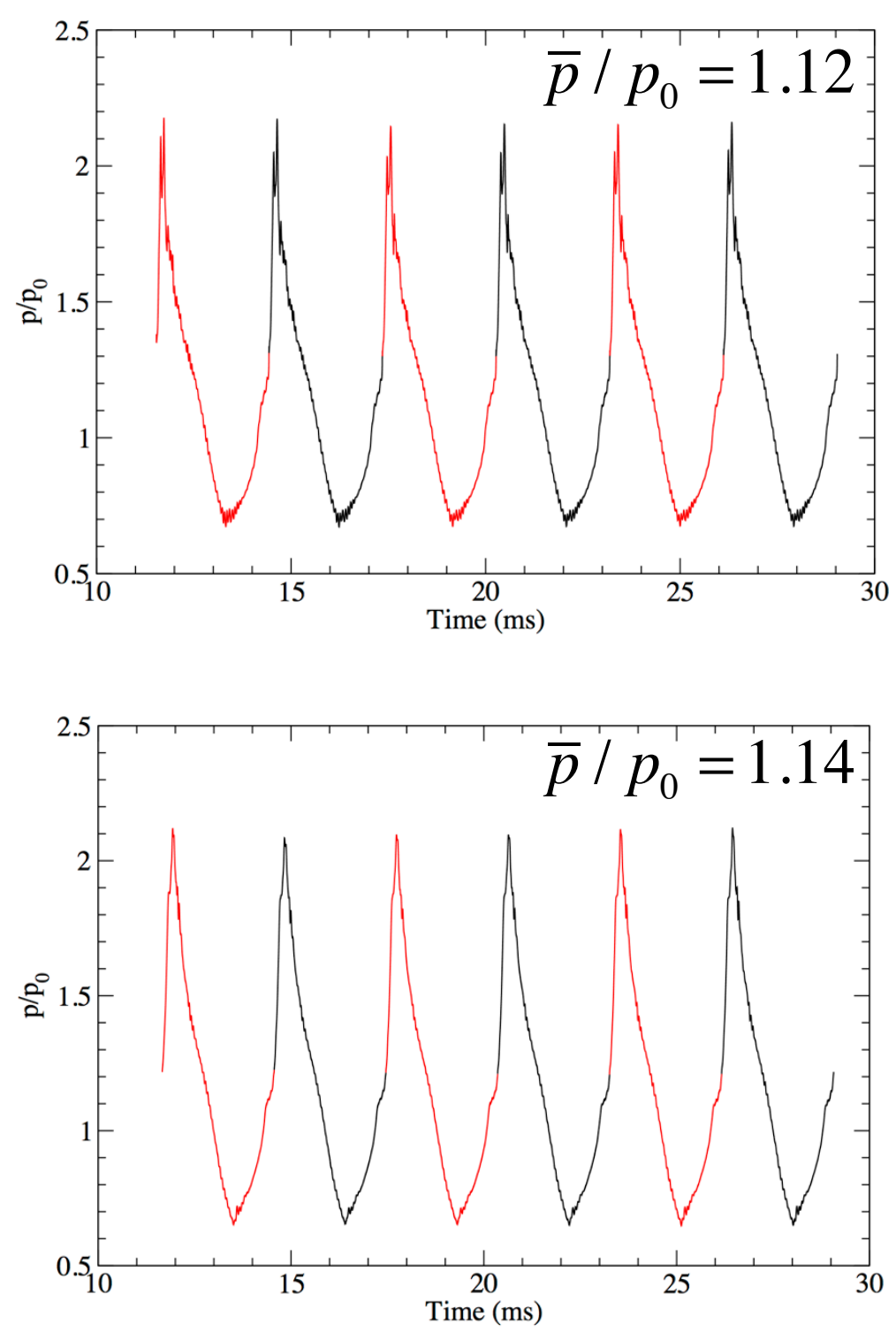

FASH combustor 


\section{Pulse-Combustor Simulations at High-Pressure \\ $p_{0}=10 \mathrm{bar}, T_{0}=550 \mathrm{~K}, \Phi=0.72$}

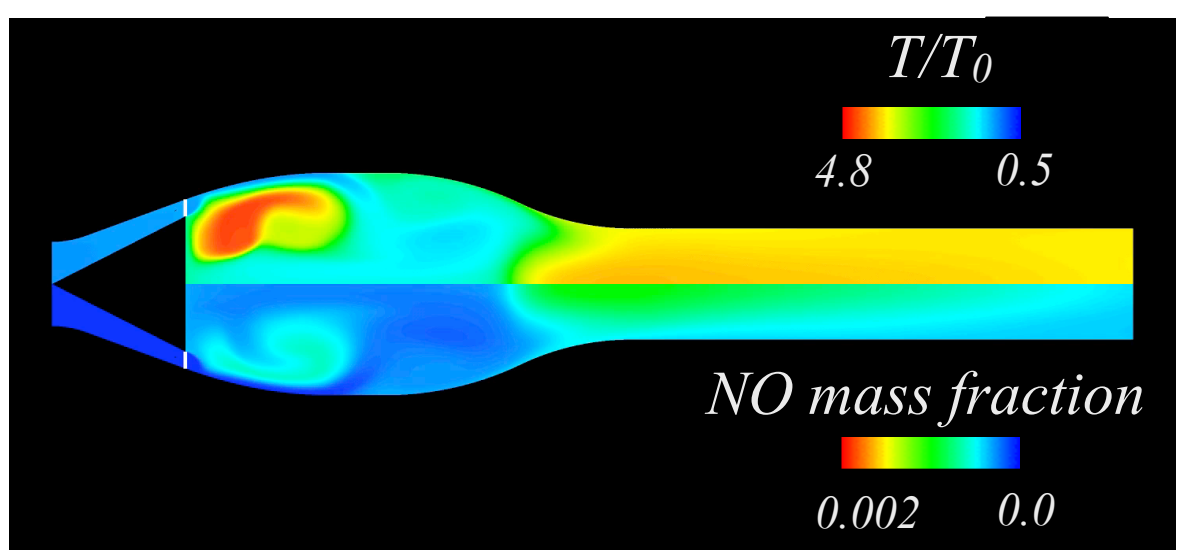

Baseline

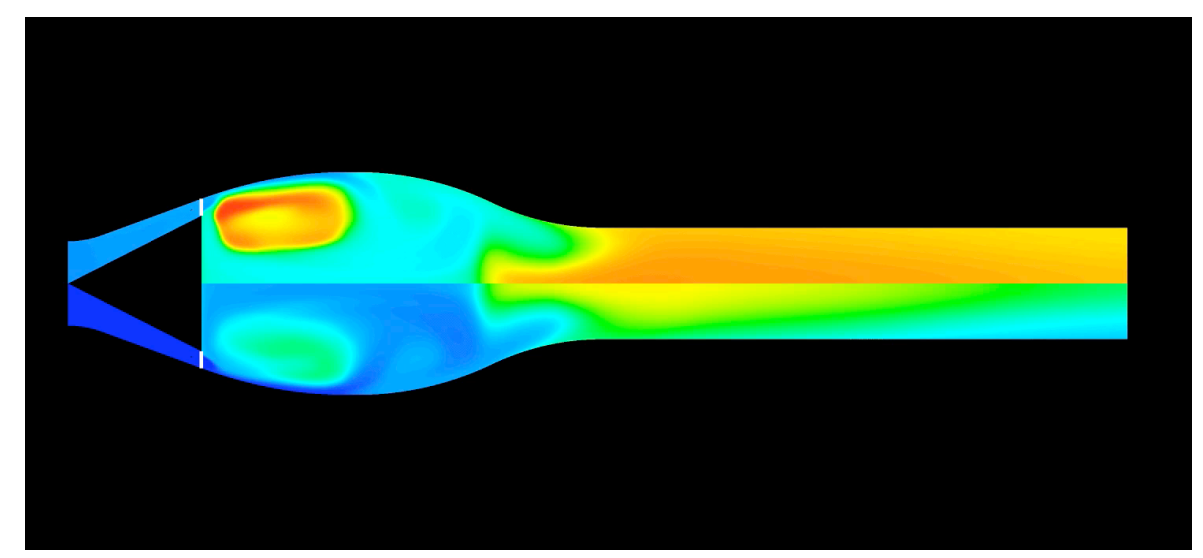

$10 \%$ shorter combustor

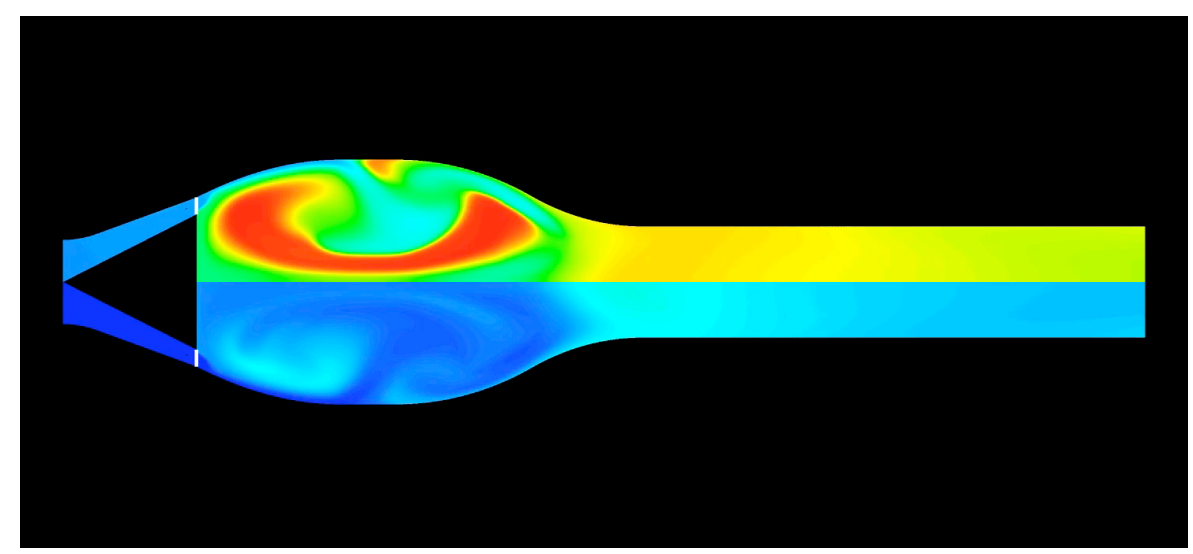

$10 \%$ larger combustor diameter

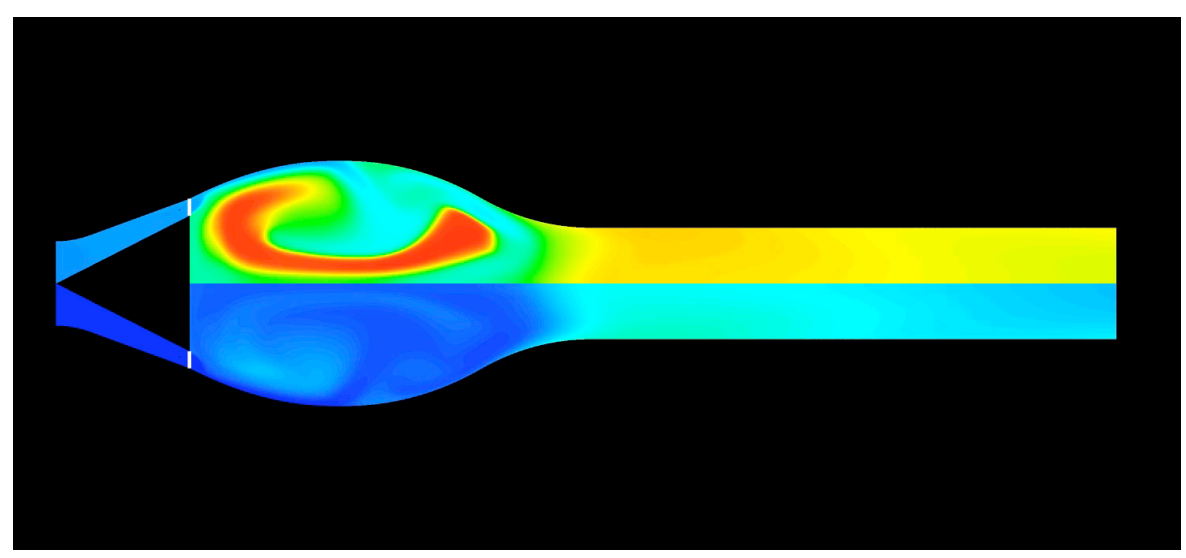

FASH combustor 


\section{OAI}

\section{Emission Index}

$$
p_{0}=10 \text { bar, } T_{0}=550 \mathrm{~K}, \Phi=0.72
$$
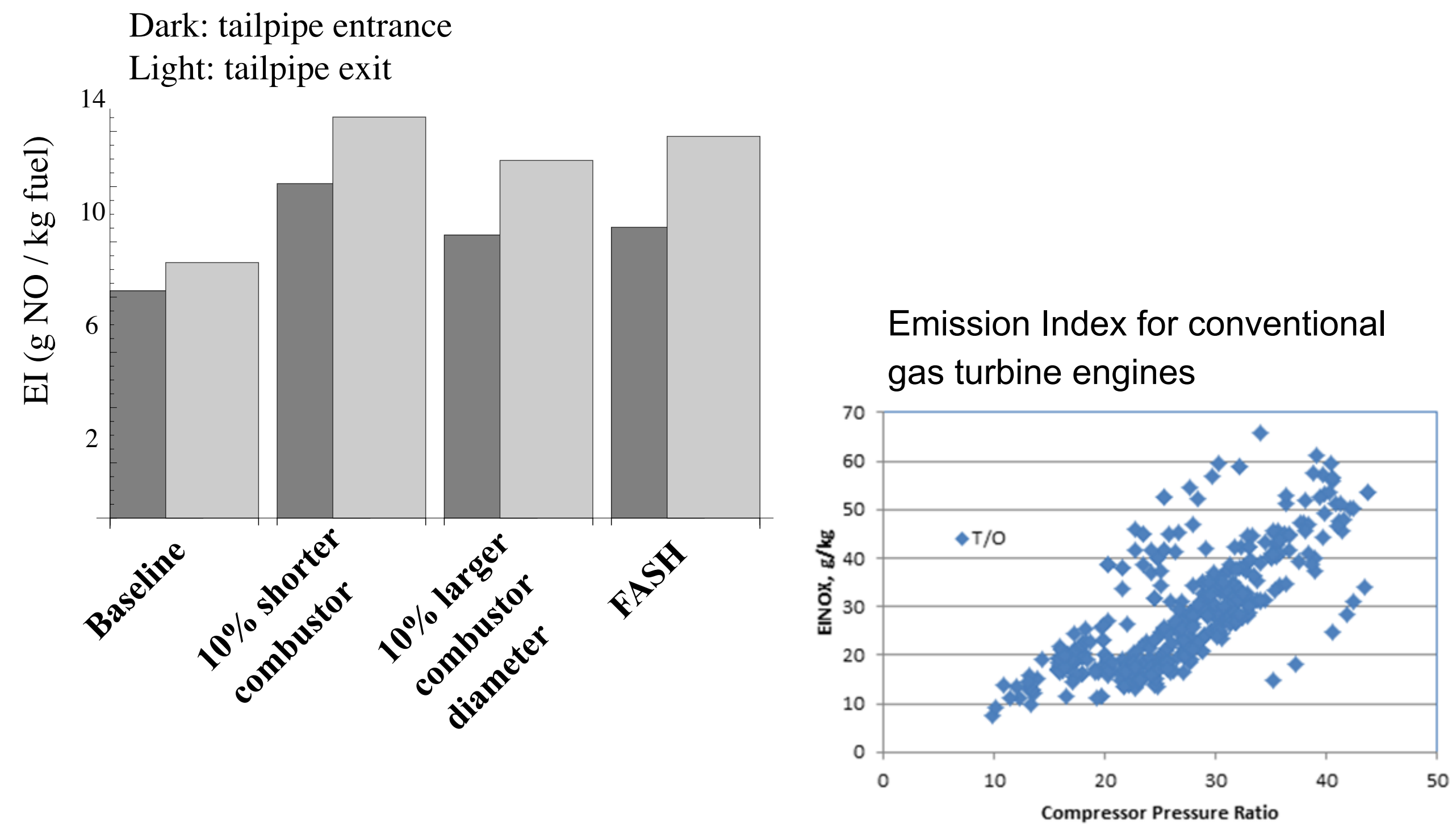


\section{OAI PES combustor based on the FASH configuration}

$$
p_{0}=10 \mathrm{bar}, T_{0}=550 \mathrm{~K}, \Phi=0.67
$$

$$
\mathrm{f}=353 \mathrm{~Hz}
$$

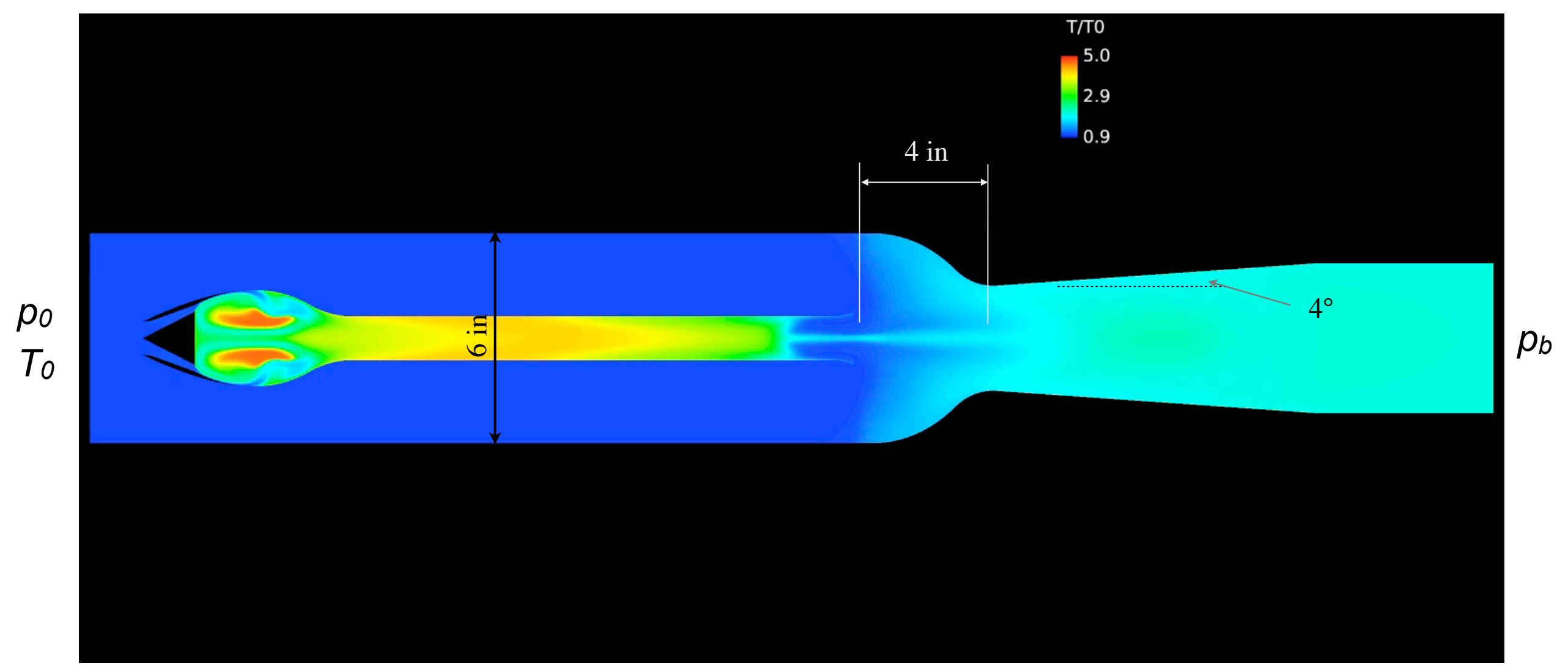




\section{OAI PES combustor based on the FASH configuration}

$$
p_{0}=10 \mathrm{bar}, T_{0}=550 \mathrm{~K}, \Phi=0.67
$$
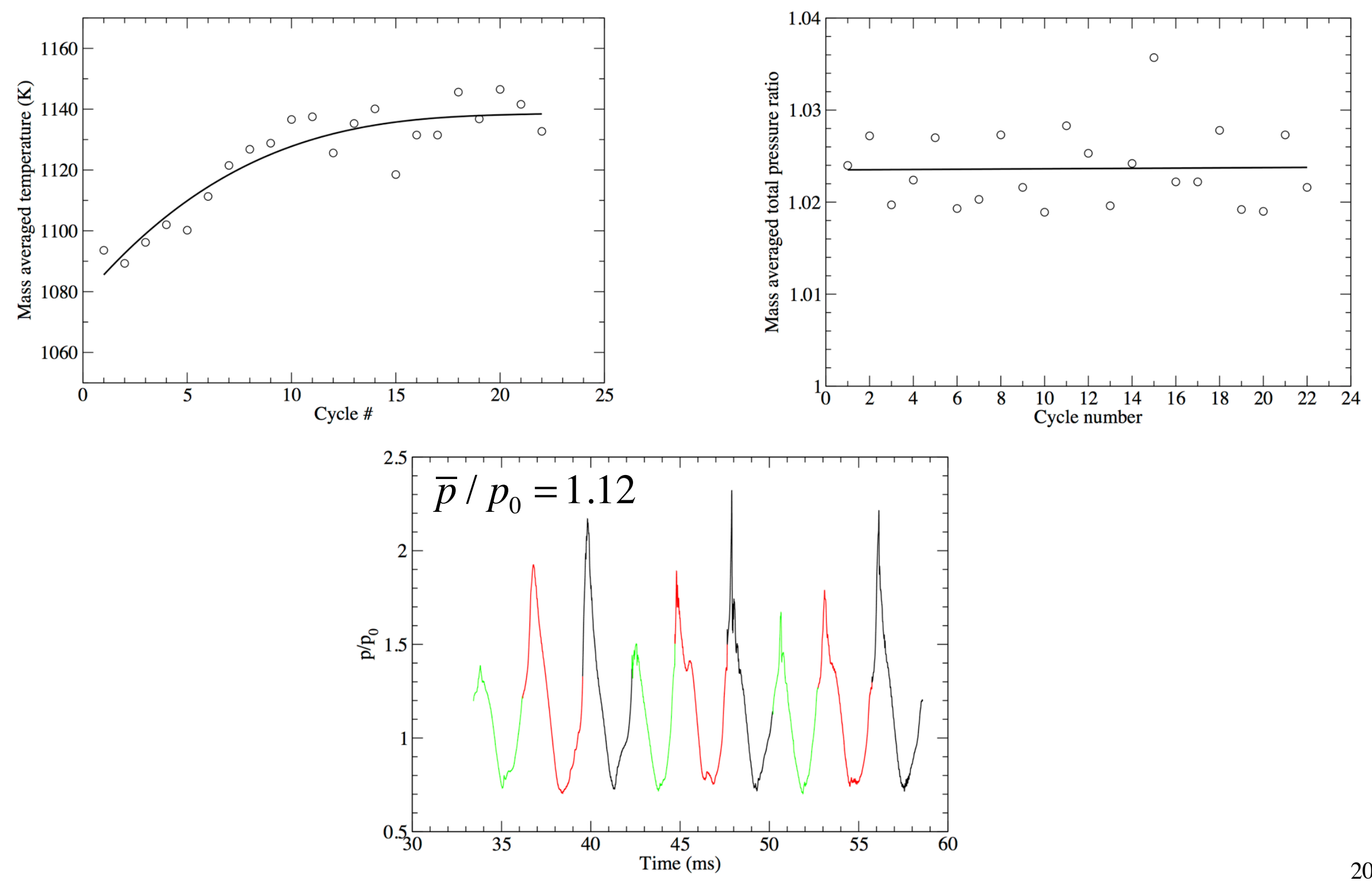


\section{PES combustor based on the Baseline configuration}
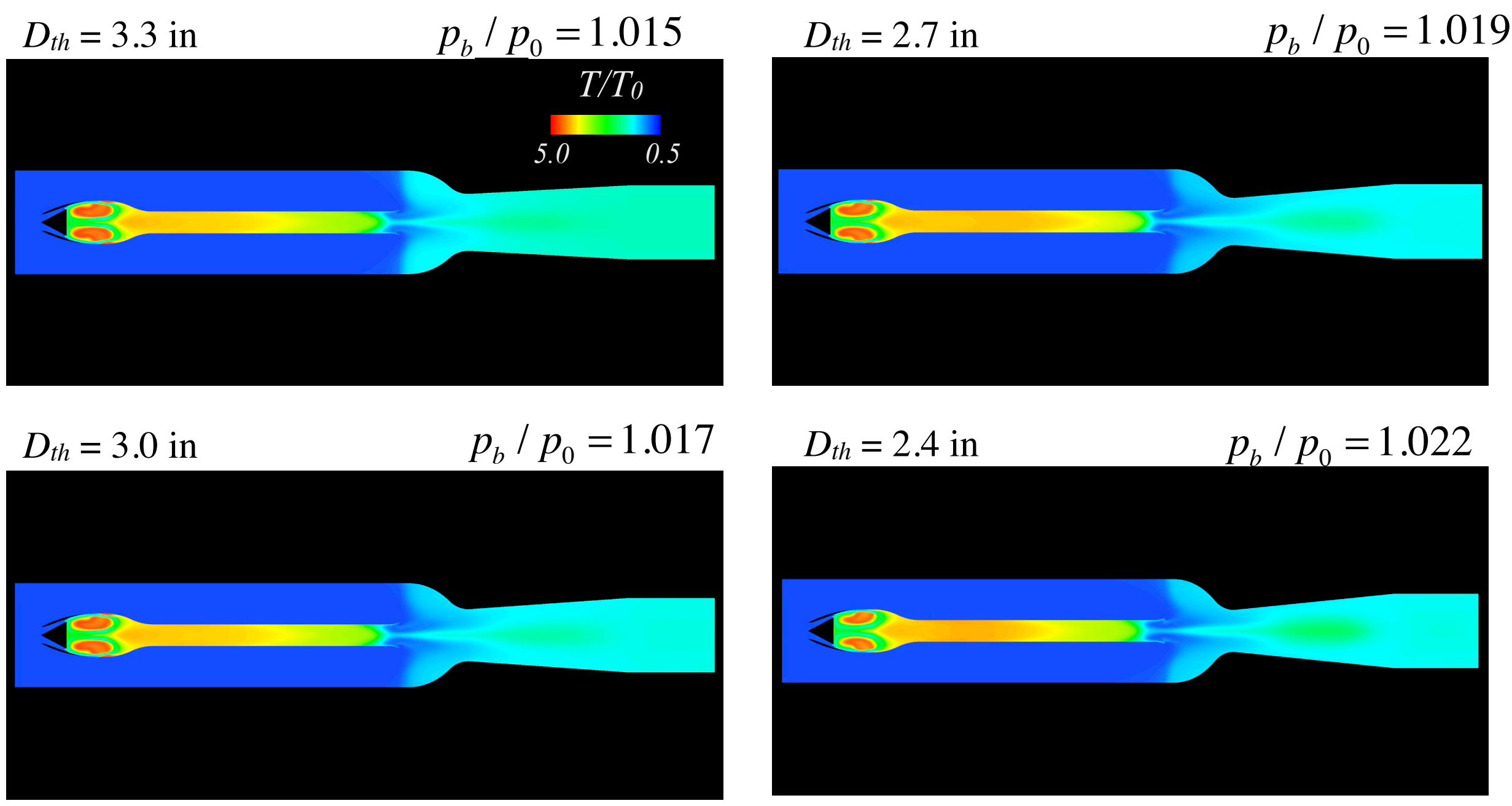


\section{PES combustor based on the Baseline configuration}

$p_{0}=10 \mathrm{bar}, T_{0}=550 \mathrm{~K}$
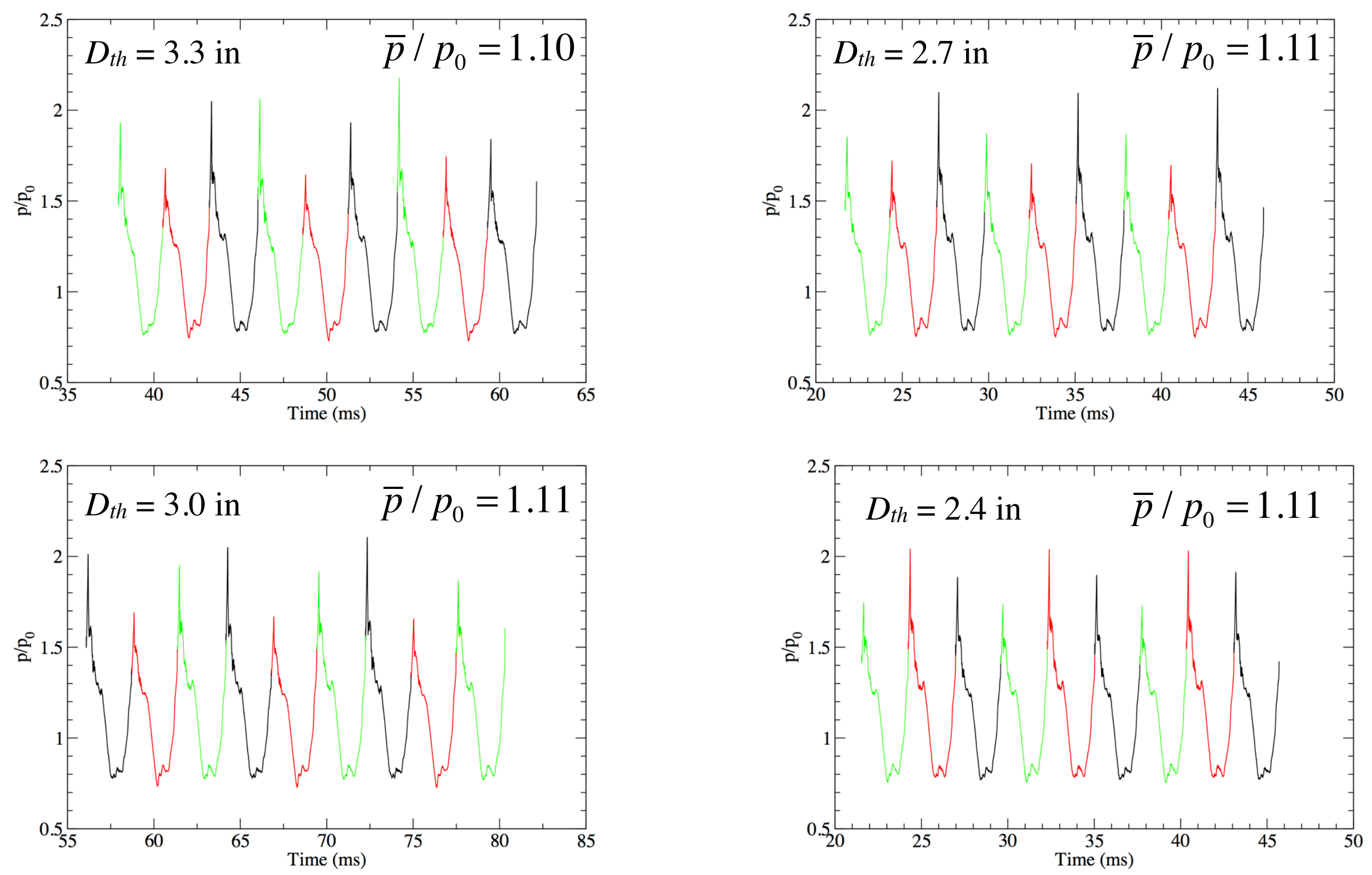


\section{PES combustor based on the Baseline configuration $p_{0}=10 \mathrm{bar}, T_{0}=550 \mathrm{~K}$}

$D_{t h}=2.4$ in

$p_{b} / p_{0}=1.022$

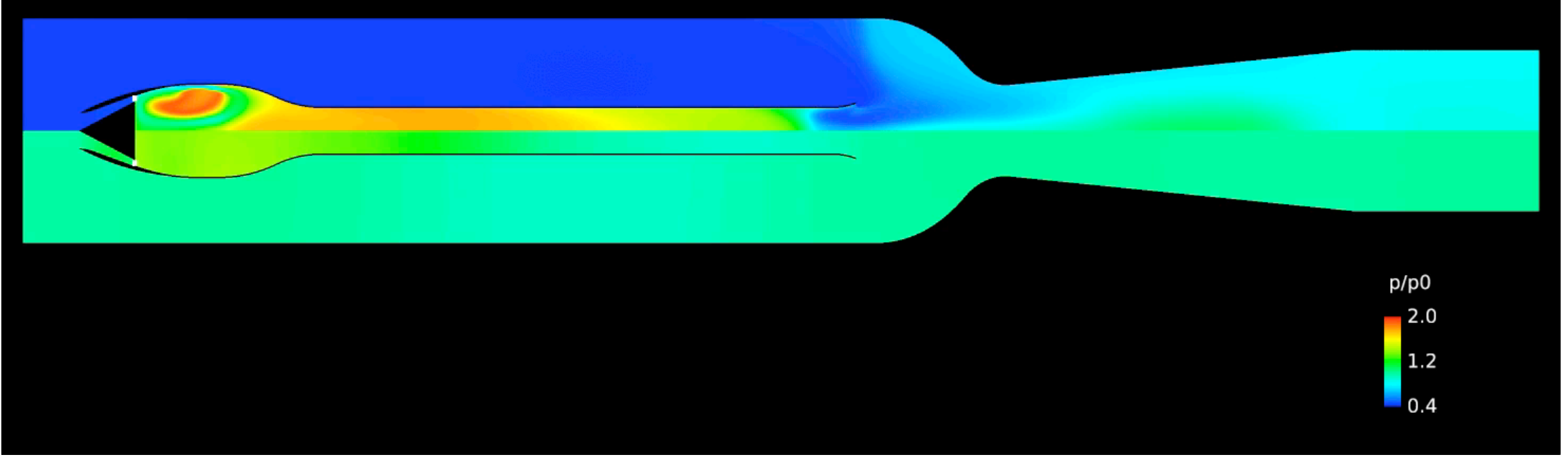




\section{PES combustor based on the Baseline configuration $p_{0}=10 \mathrm{bar}, T_{0}=550 \mathrm{~K}$}
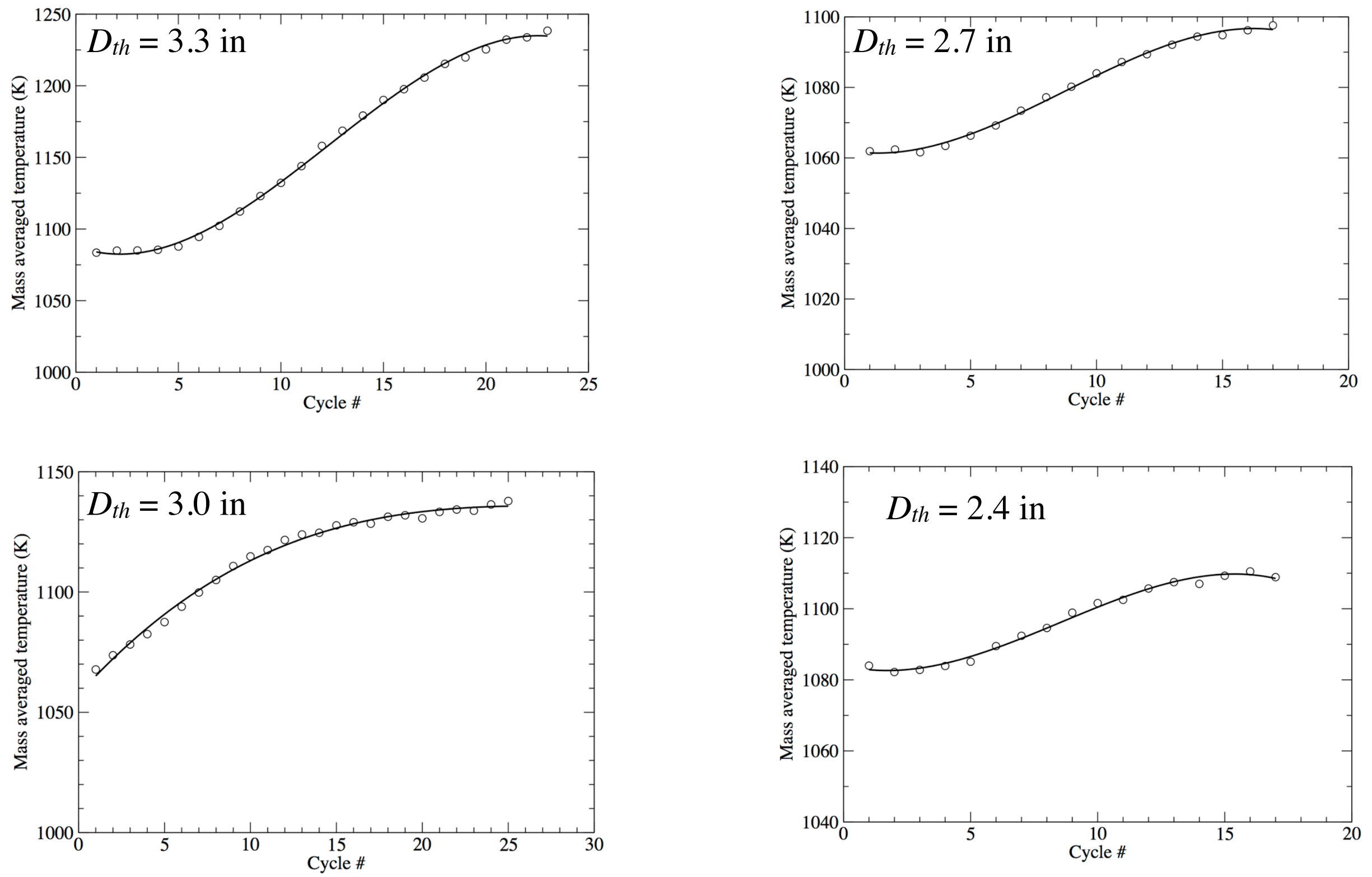


\section{PES combustor based on the Baseline configuration}

$$
p_{0}=10 \mathrm{bar}, T_{0}=550 \mathrm{~K}
$$
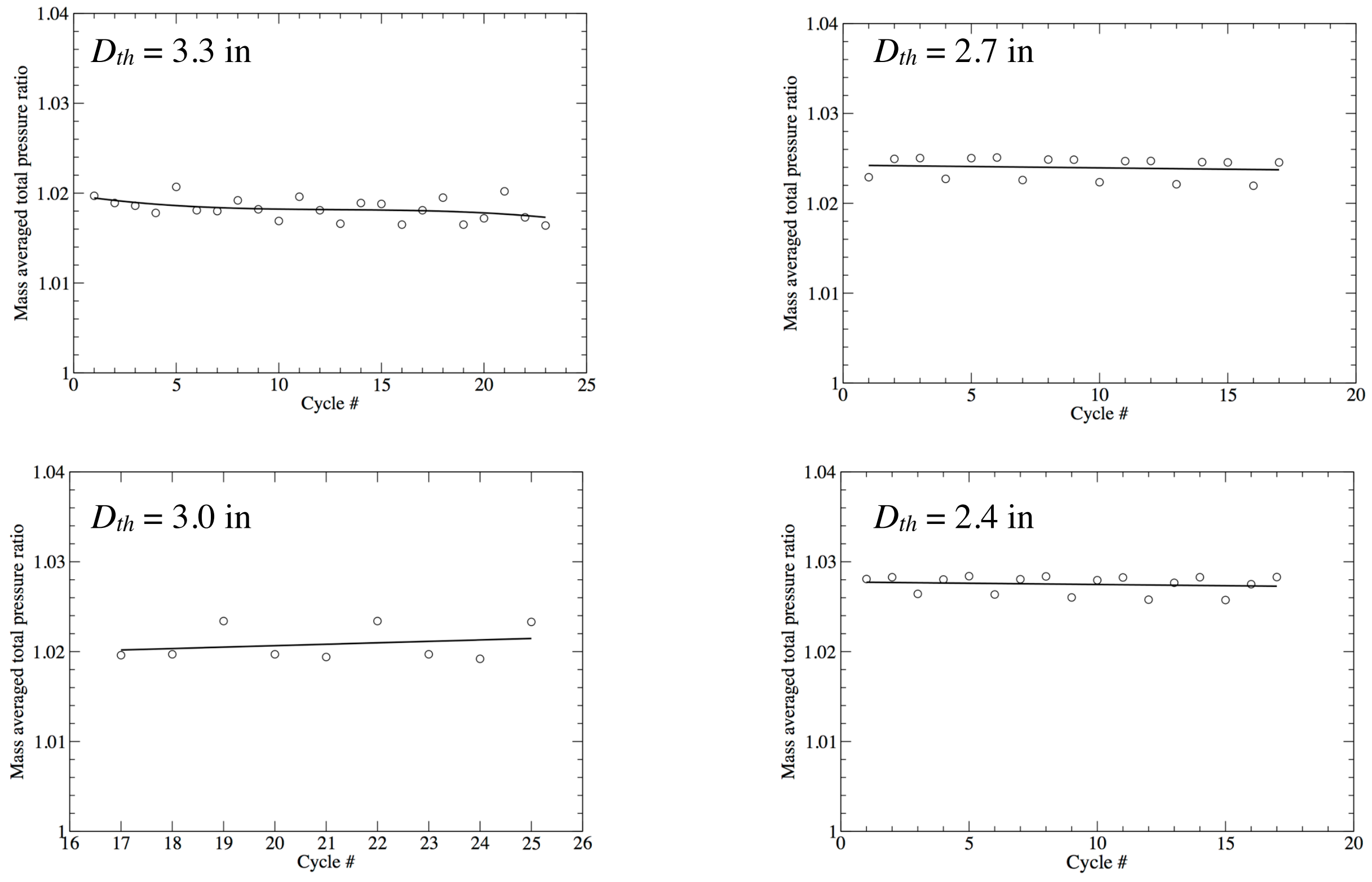
- The first part of this study analyzed new pulse-combustor configurations that were aimed at improving the fuel distribution in the pulse-combustor.

- The new configurations produced higher average combustor pressures.

- The higher pressures, however, were achieved at the cost of higher NO production.

- The emission index levels were comparable to those achieved in conventional gas turbine engines.

- The performance of various pulse-combustor driven ejector configurations were investigated computationally, focusing on the effects of ejector throat area.

- The pressure gain of the PES combustor configuration increased inversely proportional $A_{t h}$.

- The highest pressure gain achieved was $2.8 \%$, while maintaining the NOx EI $<10$.

\section{Future Work}

- Based on the results presented, higher pressure gains are likely achievable by combining the FASH-based PES combustor with the 2.4 in throat diameter ejector.

- The optimal ejector throat area and its location relative to the pulse-combustor has not yet been determined.

- Further performance improvements can potentially be achieved by improving the valve and inlet configurations to minimize pressure losses.

- New configurations currently being tested completely decouple the valve dynamics from the fuel injection process, allowing for further optimization of the fuel injection timing. 